\title{
Chemistry of Phosphorene: Synthesis, Functionalization and Biomedical Applications in an Update Review
}

\author{
Monica Pica ${ }^{1, *(1)}$ and Roberto D'Amato ${ }^{2}$ (D) \\ 1 Department of Pharmaceutical Sciences, University of Perugia, Via del Liceo 1, 06123 Perugia, Italy \\ 2 Department of Chemistry, Biology and Biotechnologies, University of Perugia, Via Elce di Sotto 8, \\ 06123 Perugia, Italy; roberto.damato1@outlook.it \\ * Correspondence: monica.pica@unipg.it
}

Received: 28 February 2020; Accepted: 15 April 2020; Published: 20 April 2020

\begin{abstract}
The present review aims to highlight the potential of an emerging 2D single element material: phosphorene. Attention is focused on the more recent studies on phosphorene, in terms of synthetic approaches, modification aimed at its stabilization, and potential applications in the biomedical field. Critical aspects for a practical use of phosphorene are discussed, in order to show a realistic scenario and challenges facing researchers.
\end{abstract}

Keywords: phosphorene; layered compounds; phosphorene stabilization and functionalization; phosphorene biomedical applications

\section{Introduction}

Graphene is the most popular 2D material consisting of a single element, exhibiting exceptional electronic, carrier transport and mechanical properties that are revolutionizing many technological areas and stimulating and inspiring the research activity of many scientists all over the world, although the lack of a band gap limits its use in electronic devices.

Another 2D single element material derived from phosphorus is emerging as a competitor of graphene, due to its promising p-type semiconducting properties, which are attracting interest for their potential applications in optoelectonics and semiconductor-based devices. Phosphorus has four allotropes: white, red, violet, and black. Among them, black phosphorus (BP) is made of layers held together by weakly attracting van der Waals forces; thus, it can be used as a source to produce freestanding 2D nanosheets, known by the name of phosphorene [1-12].

Interest in phosphorene has grown greatly in the last few years, thus expanding the perspective of applications in several different fields.

The aim of this review is to gather together the latest studies on phosphorene. Due to the high number of reviews on this topic [1-12], it was decided that attention would be paid mainly to publications from the last two years, in order to give a useful update for researchers dedicated to the chemistry of phosphorene, without neglecting significant previous works. First, the synthetic aspects will be addressed, and then, the strategies for functionalization and stabilization of phosphorene will be discussed, due to their importance for its practical use. Finally, as an inorganic chemist in a pharmaceutical science department who is well aware of the contribution of inorganic chemistry in biomedicine, it was decided to focus on the potential use of phosphorene in biomedical applications.

Before discussing the properties and reactivity of phosphorene, let's first look at its precursor, black phosphorus (BP). As reported by Bridgman in 1914, "Black phosphorus was discovered during an attempt to force ordinary white phosphorus to change into red phosphorus by the application of high 
hydrostatic pressure, at a temperature below that at which the transformation runs with appreciable velocity at atmospheric pressure [13]." Bridgman also measured the density of BP, finding a value $15 \%$ higher than that of the densest variety of red phosphorus. This result led Bridgman to conclude that BP was a new modification of phosphorus, "quite distinct from red phosphorus and, because of its higher density, presumably a more stable form". He also reported that, differently from red and white phosphorus, BP is a fairly good electrical and thermal conductor, "does not catch fire spontaneously, can be ignited with difficulty with a match, and may be heated to perhaps $400^{\circ} \mathrm{C}$ in the air without spontaneous ignition."

BP was the first single element layered material with semiconducting properties beyond those of group IV (graphene, silicene etc.). The crystal structure of BP was first proposed by Hultgren et al. in 1935 [14] and subsequently refined by Brown and Rundqvist in 1965 [15]. BP has an orthorhombic crystalline structure. $\mathrm{P}$ atoms have a $\mathrm{sp}^{3}$ hybridization, and each of them is connected to three adjacent atoms (the average bond length is $2.18 \AA$ ), with one lone pair of electrons and forming a puckered honeycomb structure (Figure 1) [1,16].

(a)
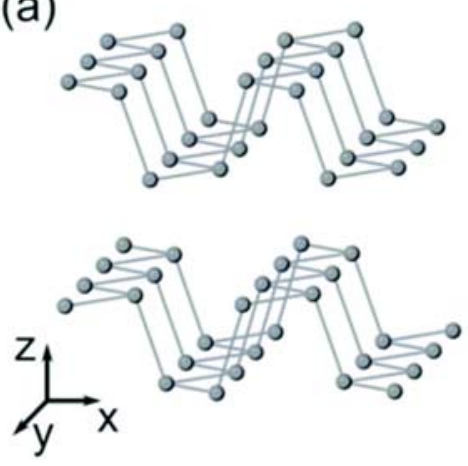

(b)

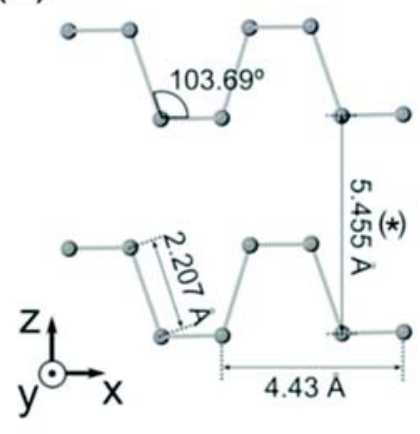

(c)

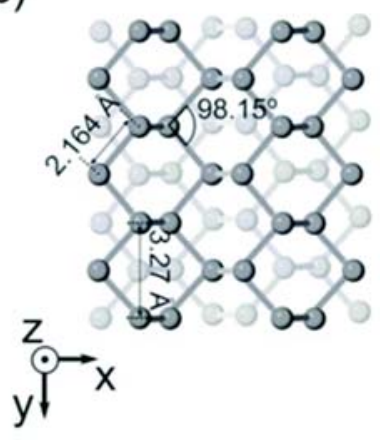

Figure 1. Structure of black phosphorus (BP). (a) 3D representation; (b) Lateral view; (c) Top view. (Reproduced with permission from [8], published by Royal Society of Chemistry, 2019).

Two of the three adjacent phosphorus atoms lie in the same plane, with an angle of $98^{\circ}$, while the third $\mathrm{P}$ atom lies at an angle of $103^{\circ}$ between the layers, resulting in various anisotropic physical properties such as optical, mechanical, thermoelectric, and electrical conductance. Adjacent layers interact via weak van der Waals interactions along the $z$ direction and stack with an ABA stacking order. The distance between two layers is about $5 \AA$ [8].

Moreover, its band gap is highly tunable, depending on doping, functionalization, and number of layers, from $\approx 1.5 \mathrm{eV}$ for a monolayer of phosphorene to $0.3 \mathrm{eV}$ for bulk BP [17]; this versatility is promising for several photoresponse devices, from the visible to the mid-infrared region.

The exfoliation of the bulk BP material, using a physical or chemical method, results in a few layers or even a monolayer of phosphorene and, as typically observed for exfoliated 2D bulk materials, the physico-chemical properties of the materials change dramatically as a result of the exfoliation.

An interesting paper by Borsacchi et al. identified and quantified the ${ }^{31} \mathrm{P}$ nuclear spin interactions determining the Nuclear Magnetic Resonance (NMR) properties of phosphorene and solid BP [18]. First, they acquired ${ }^{31}$ P Magic Angle Spin (MAS) NMR spectra of samples of phosphorene of different nanoflake size and suspended in different solvents: dimethylsulfoxide (DMSO), tetrahydrofuran (THF), or methanol. Then, a thorough analysis of the ${ }^{31} \mathrm{P}$ spin interactions was carried out on solid BP and phosphorene suspensions using solid-state NMR techniques. As far as phosphorene sheets are concerned, they found that the sheet size and the kind of solvent did not substantially affect the NMR spectra. Moreover, the extent of the main nuclear spin interactions (homonuclear dipolar coupling and shielding) determining the ${ }^{31} \mathrm{P}$ spectra of solid BP and phosphorene sheets in suspension are substantially the same. 
The properties of phosphorene, in terms of stability and reactivity, depend on the synthetic approach used for its preparation via delamination of bulk BP. In the following, some recent preparation methods are reported.

\section{Preparation of Phosphorene}

\subsection{Top-Down Methods}

Preparation methods of phosphorene can be essentially divided into top-down and bottom-up methods.

Top-down methods consist of the separation of the sheets of BP into single or a few packed layers, generally achieved using mechanical or liquid exfoliation techniques. These approaches are possible because the layered structure of the corresponding bulk BP is held together by weak Van der Waals interlayer forces.

\subsubsection{Mechanical Exfoliation}

Classical mechanical exfoliation involves the separation of the 2D sheets using an adhesive tape. Single layers are transferred onto $\mathrm{Si} / \mathrm{SiO}_{2}$ substrates, then cleaned with acetone, methanol, and isopropyl alcohol, in order to remove residual scotch tape, and finally thermally treated in order to remove the residual solvent [19].

The main drawbacks of this classical approach are the low yield, the difficulty to get large-sized monolayer or few-layer BP (FLBP), due to the instability of BP in the presence of water and oxygen and to the weak adhesive force on $\mathrm{SiO}_{2} / \mathrm{Si}$ substrates, compared with that of graphene.

Very recently, Sha et al. found that a gold or silver thin layer on the $\mathrm{SiO}_{2} / \mathrm{Si}$ substrates can significantly improve the yield and lateral size of the exfoliated FLBPs [19]. A schematic representation of their approach is shown in Figure 2.

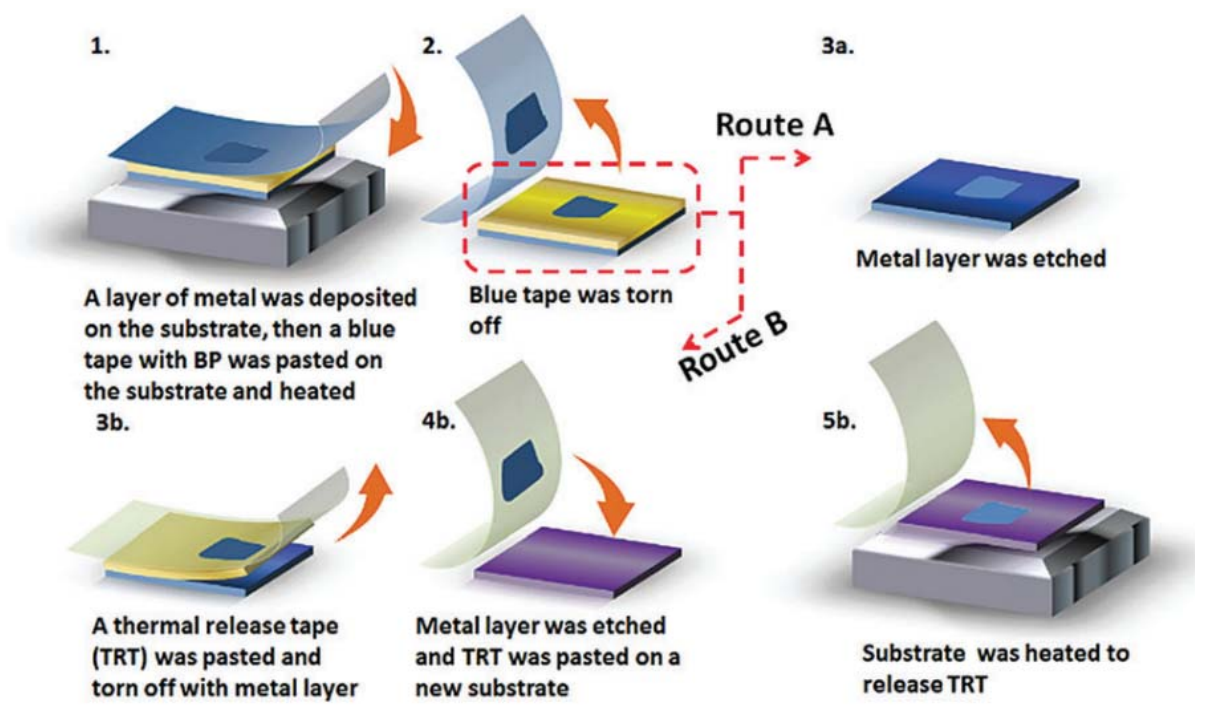

Figure 2. Illustration of the metal-assisted exfoliation process for few-layer BP (FLBP) (Reproduced with permission from [19], published by Royal Society of Chemistry, 2018).

They first deposited a gold or silver layer onto the substrate $\left(\mathrm{SiO}_{2} / \mathrm{Si}\right.$ wafer, $\mathrm{Si}$ wafer or other ones) via magnetron sputtering. Bulk BP was pasted on a blue tape and then pasted onto the substrate and heated. Then, the blue tape was torn off and the metal layer was removed so that the FLBP were left on the substrate (route A). With this technique, FLBP can also be transferred onto other substrates (route B). 
Compared with the normal "scotch-tape" method, the yield of FLBP exfoliated using the metal-assisted method is increased 100 times and the area of BP is much larger. They also proved that the exfoliated FLBP retained its morphology and crystalline structure, providing high hole mobility.

Among mechanical exfoliation methods, the plasma-assisted method is considered a promising route to fabricate monolayer or few-layer phosphorene [20].

$\mathrm{Lu}$ et al. published a paper in 2014 on the preparation of phosphorene using an $\mathrm{Ag}^{+}$plasma thinning process, by using high-quality BP crystals as starting material [21]. First, phosphorene films were exfoliated onto a $\mathrm{SiO}_{2} / \mathrm{Si}$ substrate using mechanical exfoliation and then subjected to an $\mathrm{Ar}^{+}$plasma treatment. The authors demonstrated that the experimental approach was successful in achieving stable monolayer phosphorene.

Pei et al. published a paper on the fabrication of air-stable and high-quality phosphorene samples, with a designated number of layers, through layer-by-layer thinning of thick exfoliated phosphorene flakes using oxygen plasma etching, followed by further passivation with an $\mathrm{Al}_{2} \mathrm{O}_{3}$ coating [22]. They were also able to precisely engineer the defects in a phosphorene monolayer.

The $\mathrm{O}_{2}$ plasma pre-treatment process provoked the oxidation of the top of the flake layers to $\mathrm{P}_{x} \mathrm{O}_{y}$, which acted as a protective layer for the remaining phosphorene sample underneath. The further $\mathrm{O}_{2}$ plasma etching led to the oxidation of the underlying phosphorene, so as to thin down the phosphorene layer and to increase the thickness of the $\mathrm{P}_{x} \mathrm{O}_{y}$. At the same time, the $\mathrm{O}_{2}$ plasma physically sputtered away the $\mathrm{P}_{x} \mathrm{O}_{y}$ layer from the top. Since a constant etching rate was reached, it was possible to precisely fabricate a designated number of layers of phosphorene down to a monolayer (Figure 3).

\section{a $\quad \mathrm{O}_{2}$ plasma etching}
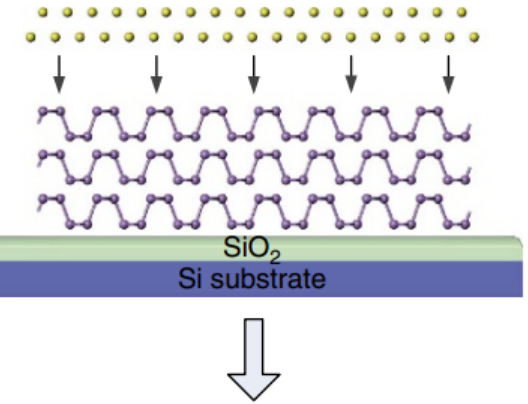

b

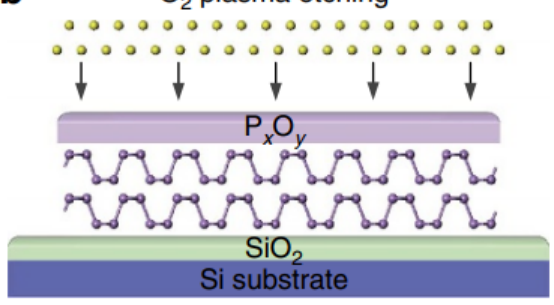

d
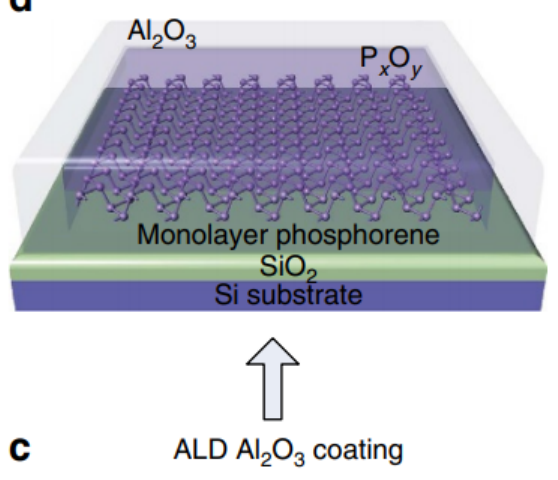

C

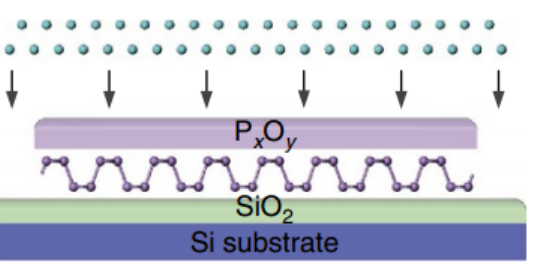

Figure 3. Schematic representation of the fabrication of air-stable mono- and few-layer phosphorene samples. (a) A thick phosphorene flake is firstly exfoliated onto a $\mathrm{SiO}_{2} / \mathrm{Si}$ substrate and the sample is then treated with $\mathrm{O}_{2}$ plasma etching (yellow balls); (b) During the $\mathrm{O}_{2}$ plasma pre-treatment process, the top layers of the phosphorene flake are oxidized to become $\mathrm{P}_{x} \mathrm{O}_{y} ;$ (c) Due to the constant etching rate, a phosphorene monolayer can be precisely fabricated and the degradation of the remaining layers is inhibited because of the protective nature of the $\mathrm{P}_{x} \mathrm{O}_{y} ;$ (d) To further improve the lifetime of the phosphorene so-produced, the sample was also coated with an $\mathrm{Al}_{2} \mathrm{O}_{3}$ protective layer [22].

S. Kuriakose et al. further studied the effects of plasma assisted layer control of $\mathrm{BP}$ in $\mathrm{Ar}$ and $\mathrm{O}_{2}$ atmospheres on the electrical and optoelectronic properties [23]. They proved that $\mathrm{O}_{2}$ plasma etching is favorable, allowing a more effective thickness control, primarily due to the formation of a surface $\mathrm{P}_{x} \mathrm{O}_{y}$ encapsulation layer. Moreover, the resultant defects can be exploited to generate Photoluminescence (PL) emissions at new wavelengths while simultaneously retaining their electrical performance. 
Yu et al. proposed a simple and efficient plasma-liquid technique for rapid ( $\sim \mathrm{min})$ and scalable production of few-layered high-quality phosphorene [24]. An anodic plasma-liquid system was used to exfoliate BP crystals to form few-layer phosphorene in pure $N, N$-dimethylformamide (DMF), without using an additional intercalator. Exfoliated phosphorene sheets in the form of a floccus precipitate were collected and the yield of the plasma exfoliation was calculated to be about $63 \%$. The phosphorene sheets had a clean and transparent surface and they were stable in DMF under ambient conditions for at least five days. The lateral size was between 1 and $10 \mu \mathrm{m}$, while the thickness of the phosphorene sheets was $4 \pm 1 \mathrm{~nm}$, corresponding to $\sim 7$ layers. Moreover, phosphorene prepared using plasma-liquid technology showed less oxidation than that prepared using the ultrasonication method, and no relevant N-P bonds were found, indicating that plasma exfoliation does not induce nitrogen doping.

Phosphorene sheets of different thickness were also prepared by changing the applied voltage. The thinner phosphorene sheets showed a lower contrast blue color with a thickness of about $1.5 \mathrm{~nm}$ ( 2 layers), while the thicker phosphorene sheets displayed a yellow-green color with a thickness of about $10 \mathrm{~nm}(\sim 16$ layers $)$.

\subsubsection{Liquid Exfoliation}

Wet methods offer, in comparison with mechanical exfoliation, several advantages, among them the superiority in obtaining a significant mass of materials as well as solution processability, scalability, stability, and an effective path to the preparation of composite materials [25].

Liquid exfoliation basically consists of the sonication, or shear mixing, of BP crystals in suitable solvents, among them N-cyclohexyl-2-pyrrolidone (CHP), dimethylformamide (DMF), dimethyl sulfoxide (DMSO), or N-methyl-2pyrrolidone (NMP) [25,26]. Among these, NMP is the most popular solvent [15], yielding the highest BP concentrations [27], although the low volatility makes its removal difficult. The main drawback of the liquid exfoliation method is that it takes a long time $(>10 \mathrm{~h})$, negatively affecting the planar size of the phosphorene sheets, both in terms of size and size homogeneity, which are important characteristics when one considers the potential applications of phosphorene, as for example in the electrochemical field.

Gómez-Pérez et al. improved the topographical homogeneity (i.e., thickness and lateral dimension) of NMP-exfoliated BP flakes after resuspension in acetone [26]. They first prepared the NMP-exfoliated few-layer BP suspension, which was dried under vacuum in order to eliminate NMP; then, the vial was refilled with acetone and finally ultrasonicated.

The typical size of the monolayers and bilayers suspended in acetone was $99.8 \pm 27.4 \mathrm{~nm}$ and $159.1 \pm 57 \mathrm{~nm}$, respectively, with a threefold improvement in terms of dimensional homogeneity with respect to the original NMP suspension. Besides the improvement of the topographical homogeneity of the phosphorene flakes, another advantage, due to the use of acetone, was the possibility to remove the solvent without the need of excess heating that could induce BP oxidation.

Yan et al. produced large phosphorene sheets using an efficient solvothermal-assisted liquid phase exfoliation method. BP flakes were mixed with acetonitrile (ACN) in an autoclave under nitrogen and then treated at $200{ }^{\circ} \mathrm{C}$ for $24 \mathrm{~h}$ [25] (Figure 4). The reaction products were sonicated for $30 \mathrm{~min}$, $60 \mathrm{~min}, 120 \mathrm{~min}$, and $240 \mathrm{~min}$, in order to obtain different layers and sizes of BP nanosheets, and finally centrifuged to recover the supernatant. 
a

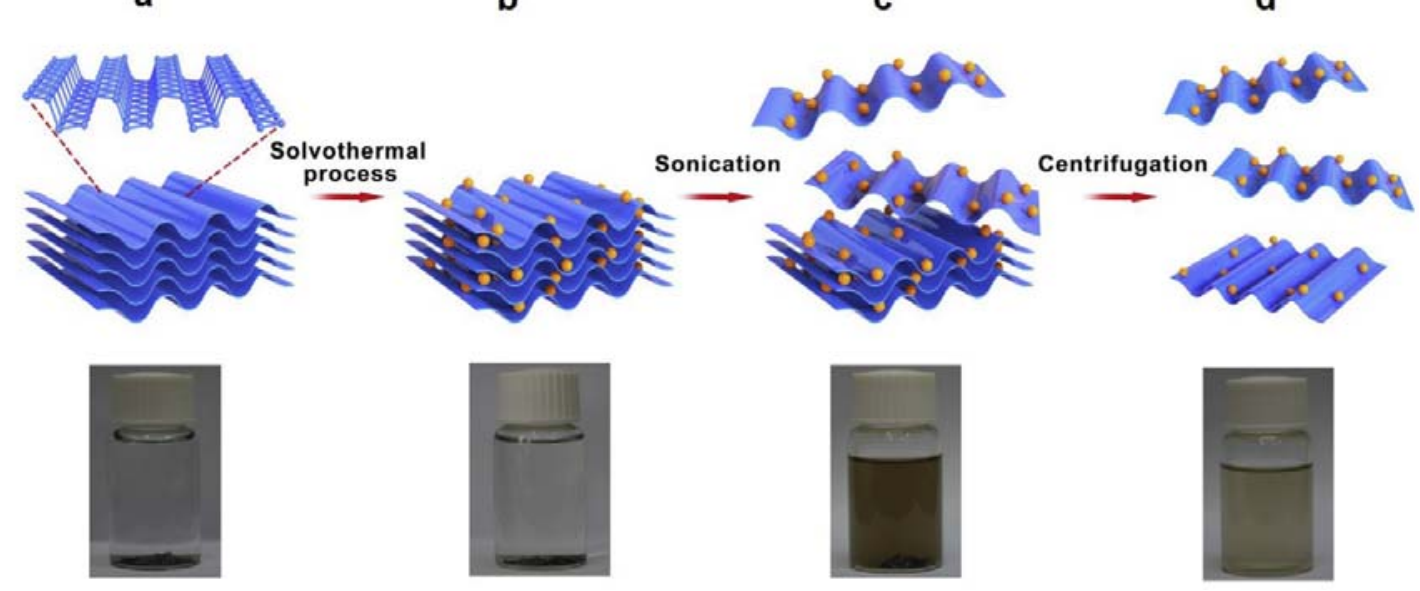

Figure 4. Schematic representation of the solvothermal-assisted exfoliation method of Yan et al. [25] and pictures of the dispersions of BP sheets in acetonitrile (ACN): bulk BP (a), insertion of ACN molecules into the interlayers of BP (b), exfoliated BP sheets dispersed in ACN (c), the obtained few-layer BP nanosheets (d) (Reproduced with permission from [25], published by Elsevier, 2018).

They found that the ultrasonic treatment for $60 \mathrm{~min}$ was the best condition to obtain large-size and high-quality BP sheets.

It was estimated that the cross-section height of the BP was in the range of 1.9-2.1 nm, corresponding to three layers of BP, while the planar size was about $10-15 \mu \mathrm{m}$.

Liu and co-workers exfoliated multilayer BP using liquid phase exfoliation in NMP mixed with saturated sodium hydroxide and absolute ethanol [28]. They prepared several samples by adding different concentrations of $\mathrm{NaOH}$, indicated with numbers from 0 (no $\mathrm{NaOH}$ ) to 3 , to a certain amount of $\mathrm{BP}$, previously ground in a glove box. The corresponding samples were indicated as MBP- $x$, with $x=0,1,2,3$. Ground BP exhibited a smooth surface, tight layer structure, uniform thickness, and neat section. MBP-2 showed a ladder shaped structure with a rough surface, non-uniform thickness, and exfoliated plackets on the edges of layers, while MBP-0 exhibited no rough surface. They inferred that the rough edge in the structure of MBP- 2 was produced by $\mathrm{NaOH}$ etching. The authors evaluated the electrochemical performance of the samples using cyclic voltammetry $(\mathrm{CV})$, charge/discharge time, and electrochemical impedance spectroscopy. The sample MBP-2 showed a large area of CV curve, long charge-discharge time, and fast charge transfer. They speculated on the role of an appropriate amount of $\mathrm{NaOH}$ in creating active sites and channels for charge storage to improve the electrochemical performance.

Watts et al. recently published a very interesting paper on the production of phosphorene nanoribbons (PNR) [29]. The method consists of a two-step process. First, macroscopic crystals of bulk black phosphorus are intercalated with lithium ions via a low-temperature, ammonia-based method. The resulting compound is then immersed in an aprotic solvent and mechanically agitated, producing stable liquid dispersions of PNRs.

They confirmed that the lithium-intercalation step is essential for ribbon production, although metal intercalation of black phosphorus is not fully understood.

They found that the process resulted in the metal atoms forming "stripes" along the corrugated channels of the black phosphorus crystal upon initial doping.

The strain between the intercalated and unintercalated regions, together with high local electron doping, caused the longer P-P bonds to break and thus cracks formed in a zigzag direction.

The ion intercalation negatively charged the layers, and when they were placed in an appropriate polar solvent, the anionic PNRs dissolved, and agitation (sonication or stirring) encouraged the individual ribbons to fully separate. 
Among the polar, aprotic solvents tested, $n$-methyl-2-pyrrolidone (NMP), dimethylacetamide (DMAc), dimethylformamide (DMF), and acetonitrile all produced ribbons, whereas tetrahydrofuran (THF) and dichloromethane (DCM) did not.

The average length of the ribbons was $690 \mathrm{~nm}$, and the average width was $30 \mathrm{~nm} ; 33 \%$ of the ribbons were narrower than $10 \mathrm{~nm}$.

The PNRs were stable in an inert atmosphere (in solution and once deposited on a substrate), but, upon exposure to air, deposited PNRs gradually oxidized, in a similar manner to 2D phosphorene.

An important aspect of this method is that it is scalable, and the stable liquid dispersions will permit the PNRs to be efficiently manipulated into thin films, membranes, composites, electrodes, heterostructures, and other devices.

\subsubsection{Electrochemical Exfoliation}

Electrochemical exfoliation is a simple, economic, and environmentally friendly approach and has been successfully used to produce 2D materials such as graphene and $\mathrm{MoS}_{2}$ [30-33].

In 2017, Pumera and coworkers first proposed the electrochemical exfoliation of BP [30]. They used an apparatus consisting of a two-electrode system, with a BP flake as the anode (electrically connected through $\mathrm{Cu}$ tape) and $\mathrm{Pt}$ foil as the cathode in acidic solution of $\mathrm{H}_{2} \mathrm{SO}_{4}$. By applying a voltage of $+3 \mathrm{~V}$, a slow release of fine material was noticed from the crystal with the solution slowly turning yellow/orange. After $2 \mathrm{~h}$, the solution turned dark orange, and after washing with pure water and drying by vacuum filtration, the exfoliated material was redispersed in DMF and sonicated for $1 \mathrm{~h}$, thus obtaining a clear green/gray dispersion.

STEM pictures showed the presence of sheets more transparent to electrons after exfoliation than those forming bulk BP, while XPS analysis highlighted that exfoliated nanosheets are more prone to oxidation, which is also favored by the anodic exfoliation process itself.

In 2018, Xiao et al. proposed a simple cathodic exfoliation of BP, conducted in a two-electrode system, where bulky BP crystal served as the cathode and Pt foil was used as the anode [34]. BP was previously expanded using tetrabutylammonium hexafluorophosphate; then, propylene carbonate was used as solvent to offer a thermodynamically adequate interface for solvation of the expanded black phosphorus. Finally, an adequate voltage was applied for a suitable time (12 h). TEM analysis proved the increased transparency for electrons, after exfoliation, in BP sheets with a thickness in the range of 2-7 nm. Interestingly, it was found that cathodic exfoliation reduces the oxidation degree of BP with respect to anodic exfoliation.

$\mathrm{Lu}$ and coworkers again proposed the cathodic exfoliation of BP for the isolation of high-quality nonoxidative few-layer phosphorene (FLBP), using tetra alkylammonium (TAA) tetrafluoroborate salts and DMSO as organic electrolyte [35]. The use of in situ optical microscopy and ex situ SEM characterization provided important information about the steps of the mechanism. It was observed that the cointercalation of the solvated TAA complex into the interlayer space of bulk BP, followed by the electrochemical decomposition of the intercalated compounds into gaseous species, created the driving force for the ultrafast expansion of bulk BP. The fully expanded BP readily dispersed in organic electrolytes to form a homogeneous dispersion. The authors also used TAA cations with different alkyl chains, ranging from methyl to octyl, to investigate the effect of the cation size on exfoliation. They found that tetrabutylammonium (TBA) gave the best results, both in terms of expansion rate (in minutes) and yield of FLBP ( $>80 \%)$. It was inferred that solvated cations, rather than the TAA cation alone, plays a key role in determining the efficiency of the intercalation and exfoliation of BP. Statistical analysis, carried out using AFM data for BP exfoliated using TBA, revealed that the average size of the exfoliated few-layer BP was $\sim 10 \mu \mathrm{m}^{2}$, while the average thickness of the exfoliated BP flakes was around $4 \mathrm{~nm}$, equivalent to five layers. Interestingly, the exfoliated BP underwent little or no oxidation and its dispersion in DMSO electrolyte displayed considerably enhanced air stability.

In a very recent paper by $\mathrm{Lu}, \mathrm{Xie}$ and coworkers, cathodic exfoliation was carried out in water using hexadecyltrimethylammonium chloride (CTAC) as intercalating agent [36]. No obvious exfoliation 
was observed at room temperature, while significant exfoliation was achieved at $50^{\circ} \mathrm{C}$. Interesting and useful SEM images were collected after the bulk BP crystals were exfoliated in CTAC solution, at $50{ }^{\circ} \mathrm{C}$, for $10 \mathrm{~min}$, applying different voltages (Figure 5).
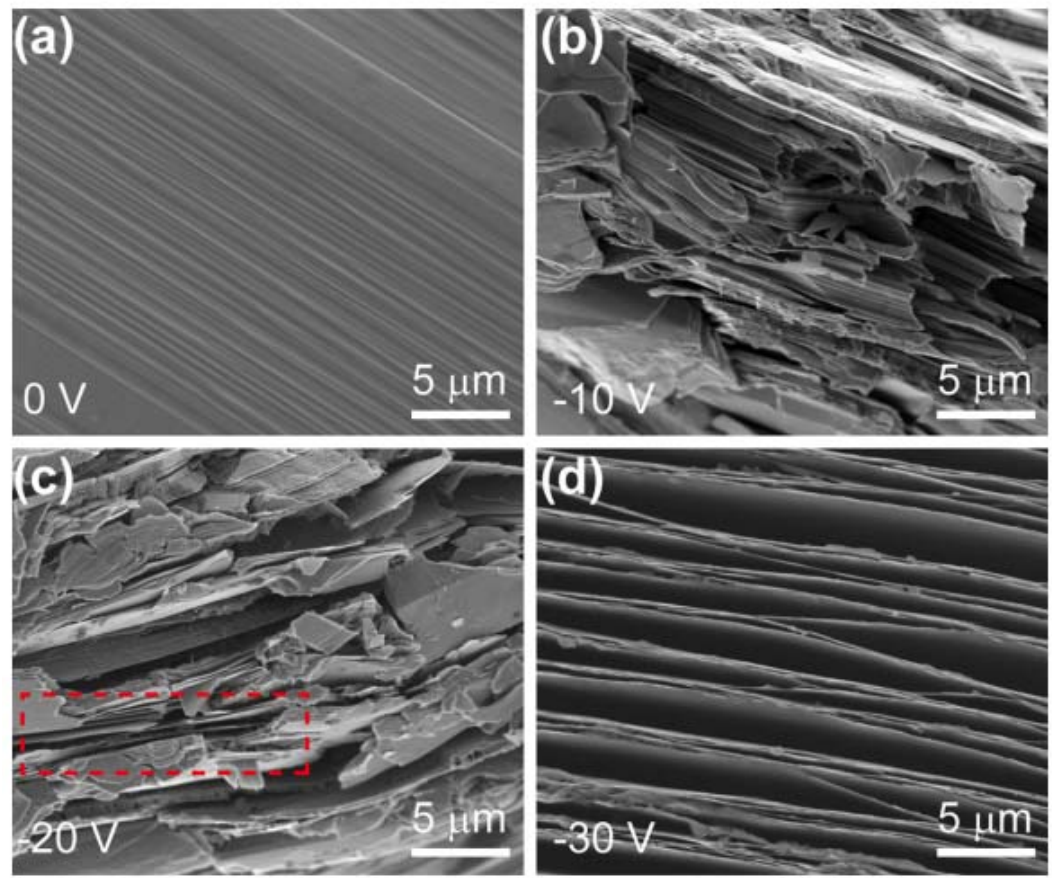

Figure 5. SEM images of the bulk BP crystals after applying different voltages at $50{ }^{\circ} \mathrm{C}:(\mathbf{a}) 0 \mathrm{~V} ;$ (b) $-10 \mathrm{~V}$; (c) $-20 \mathrm{~V}$; (d) $-30 \mathrm{~V}$ (Reproduced with permission from [36], published by American Chemical Society, 2019).

In particular, at a voltage of $-20 \mathrm{~V}$, the interlayer space, in certain parts of the treated bulk BP crystals, was slightly increased (red dashed box in Figure 5c). When a voltage of $-30 \mathrm{~V}$ was used, the interlayer space of bulk BP was dramatically increased after treatment (Figure 5d): $\mathrm{CTA}^{+}$cations can be rapidly decomposed to gaseous species at $50{ }^{\circ} \mathrm{C}$ when the voltage is lower than $\sim 25 \mathrm{~V}$.

The authors also found that the voltage needed in the above system is higher than that typically used in the nonaqueous exfoliation systems, since a higher driving force is necessary to insert CTA ${ }^{+}$ cations into the strongly interacted layers of BP in aqueous solution.

Moreover, it was also found that the $\mathrm{CTA}^{+}$cations can be adsorbed on the as-exfoliated few-layer BP flakes once they are peeled off from the bulk BP cathode. This adsorption can protect the few-layer BP flakes, preventing them from degradation.

Mei et al. prepared holey phosphorene for the first time using electrochemical assistance [37]. A holey 2D structure is characterized by nano-scaled pores on the sheets. While holey graphene has been prepared and applied in several fields, due to its higher ion transport efficiency, higher specific surface area, and interesting semiconducting properties, holey phosphorene, with respect to graphene, has only been explored using density functional theory (DFT) calculations, but has never been prepared experimentally so far due to the huge challenge in terms of preparation.

In their paper, Mei and coworkers used a sodium-ion battery with BP powders as the cathode and $\mathrm{NaClO}_{4}$ as the electrolyte. The holey material had a thinner sheet structure than BP (thickness $\approx 1-2 \mathrm{~nm}$, corresponding to ca. 1-2 layers) and exhibited a large number of holes that penetrated through the thickness of the layer, and the pore size ranged from a few to tens of nanometers, while the size of the sheet was approximately $3 \mu \mathrm{m}$ long and $2 \mu \mathrm{m}$ wide. Although the peak of the phosphorus oxide was detected using X-ray photoelectron spectroscopy (XPS), the degree of BP surface oxidation was reduced as compared with previous results [30], which indicated that this method can be used for the preparation of nano-BP with low oxidation levels. 


\subsection{Bottom-Up Methods}

Actually, there are no promising bottom-up methods for the preparation of phosphorene, especially on a large scale. Inspiration could come from the approaches typically used for graphene and transition-metal dichalcogenides (TMDs), among them hydrothermal synthesis or Chemical Vapor Deposition (CVD) growth, even though their switch to phosphorene synthesis requires significant problems to be solved.

Although CVD is quite useful for growing monolayers of TMD, graphene, etc., it is not reliable for the deposition of phosphorene, due to the absence of suitable phosphorus-based precursors, since most of them are highly toxic [38], while the main problem of hydrothermal methods is the instability of phosphorene in oxidizing conditions.

The use of boiling water in hydrothermal synthesis, as well as other wet-chemical methods like solvothermal synthesis, template assisted growth and self-assembly methods, could be successful for the synthesis of phosphorene in oxygen-free conditions [38].

Zhang et al. reported the molecular beam epitaxial growth of single layer phosphorene on $\mathrm{Au}(111)$ using black phosphorus as precursor, studied by combining in situ low temperature scanning tunneling microscopy and density functional theory calculation [39]. Specifically, phosphorus was deposited by evaporation from a crucible containing bulk black phosphorus at $260^{\circ} \mathrm{C}$. At that temperature, mainly $\mathrm{P}_{4}$ molecules are present and the initial growth stage involves the condensation of $\mathrm{P}_{4}$ molecules from the gas phase onto the $\mathrm{Au}(111)$ surface, which was heated at $230^{\circ} \mathrm{C}$ so that a phosphorus layer with larger domain size could be obtained.

Theoretical calculations predicted the key role of the substrate in the bottom-up growth of 2D phosphorene. Gao et al. reported that strong interactions between phosphorus and the substrate provoked the break of the phosphorus sheet, while weak interactions were not effective to stabilize the 2D sheet [40]. An intermediate interaction, of the order of about $0.35 \mathrm{eV}$ per $\mathrm{P}$ atom, is suitable for $2 \mathrm{D}$ phosphorene growth.

In their paper, Gao and coworkers also investigated the effect of different substrate on the growth of 2D phosphorene, by comparing the behaviors of phosphorus on $\mathrm{Cu}(111), \mathrm{Au}(111)$, and on tellurium-functionalized $\mathrm{Au}(111)$. They found that phosphorus strongly interacted with $\mathrm{Cu}(111)$, with consequent formation of randomly dispersed phosphorus nanoclusters and single atoms instead of the formation of an extended layer, which is promoted if the initial growth is carried out on a weakly interacting surface. The interfacial interaction between phosphorus and the substrate can be weakened by functionalization of $\mathrm{Au}(111)$ with monolayer tellurium. The combination of different techniques, among them low-temperature scanning tunneling microscopy (LT-STM), X-ray photoelectron spectroscopy (XPS), and density functional theory (DFT) studies, indicated the successful growth of a quasi-free-standing single-layer phosphorene on $\mathrm{Te} / \mathrm{Au}(111)$, which could enable their further application in electronic and optoelectronic devices.

In 2018, Tian et al. reported the synthesis of few-layer BP nanosheets in gram-scale quantities though a bottom-up approach, consisting of a direct solvothermal process in an ethylenediamine system using white phosphorus as raw material in the temperature range $60-140{ }^{\circ} \mathrm{C}$ (Figure 6) [41]. The materials had a 2D sheet-like structure with a lateral size of $0.8-1.0 \mu \mathrm{m}$, with the flake thickness decreasing with the increase of temperature until $100^{\circ} \mathrm{C}$. Further increasing temperature leads to the increase of flake thickness. The thickness of the BP flakes was found to be in the range of 1-15 nm, which corresponds to 2-28 atomic layers of phosphorene. Although the inevitable oxidation occurred in the samples, it was reported to be beneficial for the improvement of the stability of BP.

$\mathrm{Xu}$ et al. reported in a communication the successful molecular beam epitaxy (MBE) growth of few-layer black phosphorene quantum dots (BPQDs) directly on Si substrates at relatively low temperature using white phosphorus as the precursor [42]. AFM analysis revealed that the BPQDs had an average radius of $27.5 \pm 5 \mathrm{~nm}$ and height of $3.1 \pm 0.6 \mathrm{~nm}$. The authors also reported that after six months of ambient exposure, only phosphates were present. The BP disappeared as a consequence 
of the photoinduced oxidation and a layer-by-layer etching process after a long time exposure to ambient conditions.

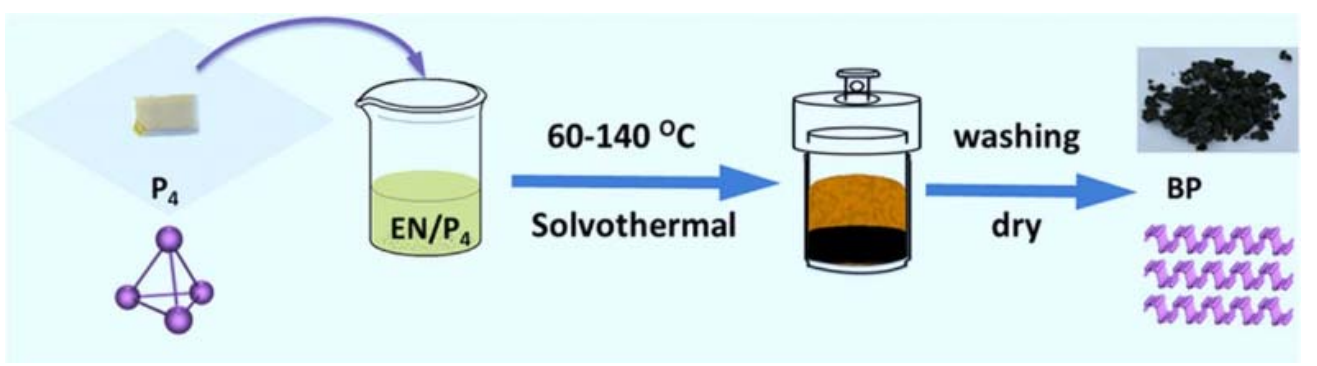

Figure 6. Schematic representation of the synthesis of few-layer BP nanosheets through a solvothermal process at the temperature range of $60-140{ }^{\circ} \mathrm{C}$ using white phosphorus as raw material and ethylenediamine (EN) as solvent [41].

\section{Functionalization of Phosphorene}

Generally speaking, functionalization of 2D materials is performed to tune physical, chemical, and electronic properties, in light of specific applications. In the case of phosphorene, functionalization is needed to improve the stability, due to its high sensitivity to water and oxygen, which react with the electron lone pair of $\mathrm{P}$ atoms. Moreover, light speeds up the degradation process of phosphorene to form $\mathrm{P}_{x} \mathrm{O}_{y}$ on the surface. The limiting step in the oxidation rate is influenced by the oxygen concentration, energy gap, and light intensity, as well as layer thickness, due to the fact that reducing the layer thickness enlarges the gap and shifts the valence band maximum and conduction band minimum to higher energies, thus matching those of oxygen $[43,44]$. Currently, the degradation process is not well understood, and its systematic study is under investigation.

Several strategies have been considered for the stabilization of phosphorene through functionalization [45], among them there is (i) the introduction of a thin oxidized protecting layer, (ii) heterostructure formation with other 2D materials, (iii) passivation using non-covalent interaction with organic molecules, (iv) encapsulation, (v) covering of the surface with acidic chemical groups, and (vi) including transition metal acceptor fragments. Depending on the kind of interaction (covalent or non-covalent) between the layer surface and the functional groups of the protecting agent, different results are obtained. Covalent functionalization could modify the intrinsic properties of phosphorene, while non-covalent modification has the advantage of avoiding degradation of the electronic structure of the original material. It was recently proved that phosphorene is able to anchor a wide type of soft materials [46] and, in this regard, an interesting book chapter on phosphorene functionalization was recently published by Ghambarian et al. [47].

Herein, we report the most recent papers dealing with the functionalization/protection of phosphorene, based on non-covalent functionalization of phosphorene.

Pumera et al. studied the non-covalent functionalization of shear exfoliated $\mathrm{BP}\left(\mathrm{BP}_{\mathrm{SE}}\right)$ nanosheets with the redox probe anthraquinone (AQ) [46]. Functionalization reaction was carried out by suspending exfoliated $\mathrm{BP}$ and $\mathrm{AQ}$ in methanol. XPS proved the presence of a carbon-based layer in the $\mathrm{BP}_{\mathrm{SE}}-\mathrm{AQ}$ sample, due to the presence of $\mathrm{AQ}$, as well as the lower oxidation percentage of phosphorus. The long-term stability of shear exfoliated BP and BP-AQ nanosheets was evaluated 30 days after their preparation. $\mathrm{BP}_{\mathrm{SE}}$ nanosheets exhibited extensive degradation with formation of bubbles deforming the nanosheet shape, due to the oxygenated phosphorus species formed in the presence of $\mathrm{H}_{2} \mathrm{O}$ and $\mathrm{O}_{2}$. On the other hand, the bubble-like features were sparse or even not observable in the $\mathrm{BP}_{\mathrm{SE}}-\mathrm{AQ}$, indicating that $\mathrm{BP}$ non-covalent modification with hydrophobic $\mathrm{AQ}$ can be advantageous to ameliorate BP degradation.

Kumar [48], for the first time, applied a simple and yet useful strategy to combine 2D phosphorene and an artificial helical copolymer (a polypetide), chemically engineered for its architecture and supramolecular assembly. The helical polymer provides several advantages: (1) it passivates and 
encapsulates BP nanosheets, (2) it electronically interacts with the BP nanosheets, (3) it can allow the fabrication of inorganic BP nanosheets for their integration in devices without sacrificing the inherent properties of BP nanosheets, and (4) it protects BP nanosheets from atmospheric oxygen. The major highlight of this research included the non-covalent passivation and encapsulation of BP nanosheets within optically active micelles or nanopockets composed of helical chains, thereby creating a shell or coating around the nanosheets while a signal could still be generated via electron transfer. These assemblies of BP nanosheets with biocompatible artificial polypeptide could be integrated with biomedical devices for suitable applications such as sensor and drug delivery.

Wu et al. passivated phosphorene with self-assembled monolayers of hexamethylenediamine (HMA) in chloroform [49]. First, they settled the levels of water and oxygen in the glove box in order to have uniform hydroxylation of phosphorene under controlled conditions. The -OH groups were introduced to promote further interaction with the ammino groups of the HMA molecules, through ionic or hydrogen bonds, so as to obtain a homogeneous protective monolayer, having thickness of approximately $1.4 \mathrm{~nm}$.

Toffanin et al. studied the interaction between boronic derivatives (acid or ester) of pyrene and BP [50]. Pyrene derivatives were selected because they are a class of largely investigated solvatochromic, conjugated, planar fluorophores, which find wide use in optoelectronic and sensing applications. $\mathrm{BP}$ was expected to show a high affinity with boron containing compounds. BP-boronic derivative adducts, both in suspension and supported on quartz substrate, were prepared. NMR spectroscopy confirmed the absence of chemical interactions between BP and boronic derivatives. Interesting results came from ageing studies. The stability of functionalized BP was evaluated after three months or six months of air exposure. The study revealed that the organic layer, acting as a physical barrier for water/oxygen and as an energy stabilizing agent, had a strong protecting function. Moreover, the intimate interaction between BP flakes and organic compounds, affecting the protection function, was much weaker in suspension than in the solid state because of the scavenging solvent molecules.

\section{Biomedical Applications}

As far as biomedical applications are concerned, 2D nanomaterials are promising in optical imaging, biosensing, phototherapy, targeted delivery, and other fields [51-57]. The tunable band gap of phosphorene, which is size-dependent, allows its interaction with the electromagnetic field to be manipulated in a layer-dependent manner, in the ultraviolet (UV) and near-infrared (NIR) regions. Its interesting optical properties make phosphorene suitable for many applications such as field-effect transistor (FET) based gas sensing and biodetection, as well as bioimaging and cancer therapy. Moreover, its puckered layer structure provides a surface area suitable for interaction with many drugs, bioactive molecules, fluorescent molecules, and metal atoms that can be loaded via nondestructive non-covalent bonds, maintaining the bioactivity of the loaded agents and allowing a number of biological applications, e.g., targeted drug delivery, biomolecule detection, cell imaging, and cancer therapy (Figure 7) [51]. Furthermore, compared to those of other common 2D nanomaterials, phosphorene exhibits a lower cytotoxicity and good biodegradability in the body [51]. All these features stimulated the interest of scientists searching for new materials with high versatile properties for multidisciplinary biomedical applications. Despite the high number of papers dealing with theoretical studies on the possible interaction of phosphorene with biomolecules, a lower number of works report real experimental results on its use in biomedical applications. In the following, some very recent papers on the use of phosphorene in various biomedical applications, such as biosensors, drug delivery, and bone regeneration, are reported. 


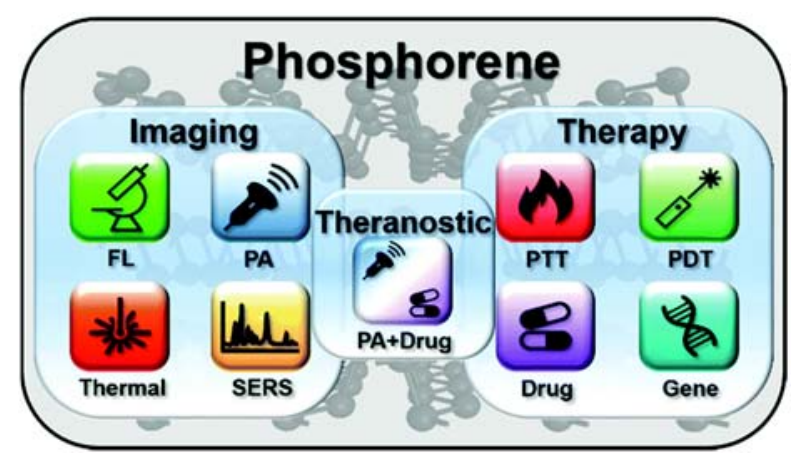

Figure 7. The multidisciplinary biomedical applications of phosphorene, including diagnostic imaging, phototherapy, therapeutic agent delivery, and theranostics (Reproduced with permission from [51], published by Royal Society of Chemistry, 2018).

Sun et al. evaluated the antibacterial properties of solvent-stabilized few-layer BP [57]. First, they prepared stable dispersions of phosphorene sheets in $N, N^{\prime}$-dimethylpropyleneurea (DMPU), using bath ultrasonication. They proved that polar aprotic solvent DMPU is more effective in exfoliating $\mathrm{BP}$ than other tested solvents, yielding approximately $16 \%$ BP nanosheet exfoliation (for an initial BP concentration of $1 \mathrm{mg} \mathrm{mL}^{-1}$ ) vs an exfoliation yield of $<8 \%$ achieved with the other solvents.

Microscopy analyses showed that the thickness values range from 2.0 to $15.4 \mathrm{~nm}$ (about $85.0 \%$ of the flakes have fewer than five layers), while the lateral dimensions were in the range $100 \mathrm{~nm}-4 \mu \mathrm{m}$. No bubbles, droplets, or other signs of BP degradation were observed. Interestingly, the phosphorene dispersion retained stability against sedimentation over a reasonably long time, even after exposure to low $\left(-5^{\circ} \mathrm{C}\right)$ or high $\left(80^{\circ} \mathrm{C}\right)$ temperatures for $12 \mathrm{~h}$, and despite 7 days of ambient exposure, the BP nanosheets had a low oxide content of ca. 4.3 atom \%.

Antimicrobial activity was tested for Gram-negative Escherichia coli (E. coli) and Gram-positive Staphylococcus aureus (S. aureus). BP nanosheets exhibited remarkable antibacterial capabilities with killing ratios against both E. coli and S. aureus as high as about $99.2 \%$, much higher than those of graphene and bulk BP (bacterial killing ratios of less than $60 \%$ ).

$\mathrm{BP}$ nanosheets have more accessible surface area than bulk BP, which favored trapping or wrapping of microbes and subsequent formation of agglomerates. More bacteria were observed to aggregate on or around BP nanosheets relatively to bulk BP and graphene, leading to enhanced antibacterial efficiency. Low cytotoxicity of BP nanosheets was also verified.

Pumera et al. fabricated enzymatic phenol biosensors consisting of 2D pnictogens, among them phosphorene [58]. Nanosheets were obtained using the shear exfoliation method, and the exfoliated materials displayed partial oxidation from the starting materials. The biosensor devices were fabricated using a layer-by-layer method of the materials, enzyme (tyrosinase), and crosslinking agent (glutaraldehyde, Glu). The obtained biosensors were used for phenol detection following $o$-quinone electroreduction to catechol, using tyrosinase (Tyr) as the enzyme responsible for the two oxidation steps of phenol to catechol and catechol to $o$-quinone. The pnictogens showed thiophilic characteristics, binding to the cysteine group of the proteins, indicating that the enzyme molecules were well immobilized onto the pnictogens. However, the phosphorene activity was similar to the control measurements (biosensor without pnictogens), indicating that, although it did not inhibit the enzymatic activity, it did not improve the electroactivity of the biosensor. The authors suggested that, among the factors responsible for the absence of activity improvement of the biosensor in the presence of phosphorene, the degree of oxidation plays an important role.

Again, Pumera, in another paper, evaluated phosphorene as an electrochemical biosensing platform in two different mediator-based oxidoreductase enzymatic systems (glucose oxidase (GOx) and peroxidase from horseradish (HRP)), to detect glucose and $\mathrm{H}_{2} \mathrm{O}_{2}$, respectively, through the reduction or oxidation of a mediator [59]. In both cases, the used mediator is the same, ferrocene methanol $(\mathrm{FcMeOH})$. BP was first exfoliated to phosphorene using sonication-assisted liquid exfoliation, using 
DMF as solvent, thus obtaining high quality phosphorene with a low level of oxidation. Phosphorene was used as the electrode in the electrochemical biosensor systems. In the first system, the flavin redox center of GOx was reduced in the presence of glucose, and the regeneration of oxidized GOx took place using ferrocenium ion $\left(\mathrm{Fc}^{+} \mathrm{MeOH}\right)$ as the mediator. Then, the interconversion of $\mathrm{Fc}^{+} \mathrm{MeOH}$ to $\mathrm{FcMeOH}$ occurred on the phosphorene electrode surface, giving rise to an oxidation signal that was related to the glucose concentration. In the second system, the HRP catalyzed the oxidation of FcMeOH to $\mathrm{Fc}^{+} \mathrm{MeOH}$ in the presence of $\mathrm{H}_{2} \mathrm{O}_{2}$, and then $\mathrm{Fc}^{+} \mathrm{MeOH}$ was rapidly electrochemically reduced at the electrode surface modified by phosphorene. Chronoamperometry measurements were performed in both systems to evaluate the ability of phosphorene as a sensing platform. The authors found that the presence of phosphorene did not enhance the recorded signal of FcMeOH oxidation with respect to that recorded without phosphorene. However, in the $\mathrm{H}_{2} \mathrm{O}_{2}$ detection, the signal recorded in the presence of phosphorene was superior to that obtained in the absence of phosphorene. In continuous oxidative potential conditions, the phosphorene was degraded, and its electron transfer capability was compromised so that in the glucose detection system the signal was low and unstable. Nevertheless, the reductive potential applied for $\mathrm{FcMeOH}$ reduction in the system based on $\mathrm{HRP}$ for $\mathrm{H}_{2} \mathrm{O}_{2}$ detection contributed to maintaining and stabilizing the BP structure.

As far as biosensors are concerned, another contribution was made by Li et al., who prepared a novel electrochemical assay for the determination of prostate specific antigen (PSA) using black phosphorene (BPene) as a platform and magnetic covalent organic frameworks $\left(\mathrm{Fe}_{3} \mathrm{O}_{4} @ \mathrm{COFs}\right)$ for signal amplification [60]. BPene was prepared via water-phase exfoliation, using sonication. Au nanoparticles were then deposited onto BPene (Au@BPene), and the nanocomposite was utilized as an immunsensing platform to bind primary antibodies and improve electron transfer. Subsequently, an Au NP loaded magnetic covalent organic framework (COF) was used to immobilize the secondary antibodies and abundant electronic signals of methylene blue (MB).

The PSA was determined using the fabricated immunosensor. The fabricated sensor exhibited linearity ranging from $0.0001 \mathrm{ng} \mathrm{mL}^{-1}$ to $10 \mathrm{ng} \mathrm{mL}^{-1}$ with a detection limit of $30 \mathrm{fg} \mathrm{mL}^{-1}$. The sensor could determine the PSA in a real sample with excellent specificity, good stability, and desirable reproducibility. The effective signal amplification of the proposed sensor was attributed to the good electron transfer of Au@BPene, excellent enrichment capacity of the signal molecules (MB) of the COF, and efficient catalytic activity of $\mathrm{Fe}_{3} \mathrm{O}_{4}$.

$\mathrm{Li}$ et al. prepared phosphorene-based $\mathrm{Au} / \mathrm{Ag}$ nanocomposites for sensitive surface-enhanced Raman scattering (SERS) detection of pesticides [61]. Phosphorene was prepared using electrochemical exfoliation using a DMF solution containing tetrabutylphosphonium bromide as the electrolyte. The characterization results demonstrated the successful synthesis of the BP/Ag/Au and BP/Au/Ag nanocomposites by the two-step process in which phosphorene served as the template and reductant simultaneously. The NPs were spread on the phosphorene surface without aggregation or pile-up. The SERS performance of the nanocomposites was evaluated using Rhodamine 6G (R6G) solutions with different concentrations. The results proved that the nanocomposites had excellent SERS characteristics, enhanced in comparison with composites containing a single type of NPs. Moreover, among the phosphorene-based $\mathrm{Au} / \mathrm{Ag}$ systems, the BP/Au/Ag nanocomposite had better SERS characteristics. To demonstrate the commercial viability of the best $\mathrm{BP} / \mathrm{Au} / \mathrm{Ag}$ nanocomposite in fungicide detection, thiabendazole and thiram were chosen as the two model molecules. For these two compounds, the US environmental protection agency (EPA) specified that the maximum residual limit (MRL) is $5 \mathrm{ppm}$. The SERS signals observed from the BP/Au/Ag nanocomposites could be detected until the concentration of thiabendazole was below $5 \times 10^{-7} \mathrm{M}$ (approximately $0.1 \mathrm{ppm}$ ), while the detection limit of thiram was $1 \times 10^{-8} \mathrm{M}$ (about $0.0024 \mathrm{ppm}$ ). Moreover, the limit of detection of thiabendazole from the $\mathrm{BP} / \mathrm{Au} / \mathrm{Ag}$ nanocomposites substrate was comparable to that obtained with other substrates in other works, while that of thiram was superior.

$\mathrm{BP}$ is also a bone constituent, in small percentages, constituting $-1 \%$ of total body weight (about $660 \mathrm{~g}$ on average) [62]. 
These properties may allow BP to be used in various applications, such as serving as a coating on scaffolds and prosthesis surfaces [63].

$\mathrm{Wu}$ and $\mathrm{Gu}$ used, for the first time, a calcium-free phosphorus strategy for enhancing bone regeneration via a BP nanosheet (BPN) hydrogel scaffold [64]. BPNs were prepared from bulk BP using a liquid exfoliation method with $N$-methylpyrrolidone, thus obtaining nanosheets with a lateral diameter from 20 to $100 \mathrm{~nm}$. Hydrogel was fabricated via photopolymerization of gelatin methacrylamide (GelMA) and cationic arginine-based unsaturated poly(ester amide)s [U-Arg-PEAs], which was incorporated into hydrogels, not only for cross-linking to form a hydrogel with improved cellular interactions, but also for the resulting positive charge that facilitated a strong electrostatic attachment of the hydrogel to BPNs, promoting a phosphate release in a sustained manner. The fabricated platform may supply phosphorus, and encapsulated BPNs can capture calcium ions to accelerate biomineralization in a bone defect.

The PEA/GelMA hydrogels showed a typical macroporous sponge-like structure with a $50 \mu \mathrm{m}$ mean pore diameter, whereas the BP/PEA/GelMA hydrogel showed a thicker wall with holes and encapsulation of BPNs, indicating a possible improvement in the network structure of hydrogels.

Photoresponsive phosphate-release testing and an in vitro mineralization experiment were also conducted. The hydrogels were immersed in pure water without phosphorus and were incubated in the dark, under natural light, or in the dark but with 5 min of near infrared (NIR) laser irradiation every $12 \mathrm{~h}$. According to the literature, BPNs can efficiently convert NIR light into thermal energy [64]. The results showed that the concentration of phosphate in the group treated with light was significantly higher than that in the group "in the dark", indicating that BPN-containing hydrogels provided extra phosphorus in a photoenergy-related manner. The mechanical properties of the BP/PEA/ GelMA hydrogels were also tested after three sessions of NIR laser irradiation within $48 \mathrm{~h}$. Compared with the hydrogels without BPNs, subjected to the same treatment, those with BPNs showed a higher compression modulus, indicating good mechanical performance with little influence on the phosphate release. To test the capacity for mineralization in vitro, the hydrogels were soaked in simulated body fluid (SBF) under natural light for 15 days. It is noteworthy that the hydrogel with the supply of calcium-free phosphorus showed obvious mineralization characteristics with a white appearance. Finally, biocompatibility tests proved the excellent biocompatibility of BP/PEA/GelMA hydrogels, which also promoted in vitro osteogenic differentiation of human dental pulp stem cells and enhanced bone regeneration in vivo.

Deng et al. used phosphorene nanosheets to prepare a system for combined photothermal and chemo cancer therapy via drug self-stabilization [65]. They used the drug itself to stabilize BP, that is the active species of platinum-based anticancer drugs (oxaliplatin, DACHPt, and cis-platin, $\left.\mathrm{Pt}\left(\mathrm{NH}_{3}\right)_{2}\right)$ were utilized to coordinate with BP nanosheets to form complex BP/DACHPt and BP/Pt( $\left(\mathrm{NH}_{3}\right)_{2}$ and improve their stability.

First, phosphorene nanosheets were prepared using liquid exfoliation of BP, using NMP as solvent. Then, platinum-based drugs were loaded onto phosphorene nanosheets twice their weight to form coordination complexes, thereby significantly boosting stability during long-term dispersion in air-exposed water and maintaining photothermal performance. BP/DACHPt with ultra-high drug loading capacity (about 200\%) underwent acid- and NIR-responsive release of intact DACHPt, thus inducing evident NIR photothermal and chemotherapeutic cytotoxicities in vitro. Finally, the $\mathrm{BP} / \mathrm{DACHPt}$ complex exerted tumor ablation in vivo based on the combined photothermal and chemotherapeutic effects.

Cui et al. realized a multifunctional drug-delivery system based on BP nanosheets (BP NS) for acute lymphoblastic leukemia (ALL) therapy [66]. BP NS were first synthesized using a liquid sonication exfoliation technique and then modified with poly ethylene glycol (PEG) through electrostatic adsorption. 
The PEG layer could effectively isolate the BP nanosheets from the outer environment to enhance their physiological stability. The PEGylated BP NS (BP NS@PEG) showed excellent biocompatibility and could load anticancer drugs (e.g., doxorubicin, DOX) with a high efficiency.

The obtained BP NS@PEG not only demonstrated excellent photothermal conversion efficiency and photothermal stability but also exhibited good $\mathrm{pH}$ and photothermal dual-responsive drug release behavior. Moreover, the BP NS@PEG were further modified with Sgc8 aptamers through covalent bonding. The aptamers provided an efficient specificity toward ALL cells and greatly increased the endocytosis of the nanocarriers in a receptor-mediated manner, which can further improve the therapeutic effect.

\section{Conclusions and Perspectives}

Phosphorene has been demonstrated to be an excellent material with potential application in several fields, among them biochemical applications. Despite its highly interesting properties, a lot of work is still awaiting researchers to make graphene a realistic graphene competitor. First, the development of cheap, easy and high yield synthetic strategies is needed for the production of large quantities of few-layer phosphorene sheets, with controlled size and morphology. To date, two of the most promising methods to realize large-scale exfoliation of BP are the electrochemical exfoliation and the shear exfoliation methods, due to their efficiency and scalability [56].

Another fundamental aspect is the stabilization of phosphorene sheets towards water and oxygen, in order to realize applicative systems with acceptable stability during their preparation and use.

As far as biomedical applications of phosphorene are concerned, one of the main and most promising biomedical applications is as a biosensing substance to sensitively detect disease. Recent studies highlighted the potential of phosphorene in bone regeneration, as well as drug carrier in cancer therapies. However, there is still a major knowledge gap in the transition from research to clinical application.

From a general point of view, if one considers biomedical applications of BP, one should consider that biological systems are comprised of different biological molecules, structures, cells, tissue or organ systems, and organisms and thus form a complex biological interface that interacts with BP and, depending on the degree of complexity of the biological component's contact area, several BP-bio interfaces are possible [51].

The rapid degradation process of BP in aqueous environments, which not only compromises its functions, but also lead to adverse effects, is another sensitive aspect. Although the instability of BP could be beneficial because of its rapid clearance and low biopersistence in biomedical applications, the oxidation and dissolution process should be controlled in a way that reduces toxicity [51].

Therefore, the comprehension of the relationship between BP's physicochemical properties and its biological activity is first of all necessary for realistic employment of phosphorene in real biomedical systems.

Frontiers are open to multidisciplinary research to take full advantage of the peculiar and fascinating properties of phosphorene.

Funding: This research was funded by MIUR within the AMIS and DELPHI projects through the program "Dipartimenti di Eccellenza 2018-2022".

Conflicts of Interest: The authors declare no conflict of interest.

\section{References}

1. Kou, L.; Chen, C.; Smith, S.C. Phosphorene: Fabrication, properties, and applications. J. Phys. Chem. Lett. 2015, 6, 2794-2805. [CrossRef]

2. Bagheri, S.; Mansouri, N.; Aghaie, E. Phosphorene: A new competitor for graphene. Int. J. Hydrogen Energy 2016, 41, 4085-4095. [CrossRef] 
3. Batmunkh, M.; Bat-Erdene, M.; Shapter, J.G. Phosphorene and phosphorene-based materials-Prospects for future applications. Adv. Mater. 2016, 28, 8586-8617. [CrossRef]

4. Liu, H.; Neal, A.T.; Zhu, Z.; Luo, Z.; Xu, X.; Tomanek, D.; Ye, P.D. Phosphorene: An unexplored 2D semiconductor with a high hole mobility. ACS Nano 2014, 8, 4033-4041. [CrossRef] [PubMed]

5. Balendhran, S.; Walia, S.; Nili, H.; Sriram, S.; Bhaskaran, M. Elemental analogues of graphene: Silicene, germanene, stanene, and phosphorene. Small 2015, 11, 640-652. [CrossRef] [PubMed]

6. Smith, J.B.; Hagaman, D.; Ji, H.-F. Growth of 2D black phosphorus film from chemical vapor deposition. Nanotechnology 2016, 27, 215602-215610. [CrossRef]

7. Chaves, A.; Ji, W.; Maassen, J.; Dumitrică, T.; Low, T. Theoretical overview of black phosphorus. In $2 D$ Materials: Properties and Devices; Avouris, P., Heinz, T., Low, T., Eds.; Cambridge University Press: Cambridge, UK, 2017; pp. 381-412.

8. Xu, Y.; Shi, Z.; Shi, X.; Zhang, K.; Zhang, H. Recent progress in black phosphorus and black-phosphorusanalogue materials: Properties, synthesis and applications. Nanoscale 2019, 11, 14491. [CrossRef] [PubMed]

9. Carvalho, A.; Wang, M.; Zhu, X.; Rodin, A.S.; Su, H.; Neto, A.H.C. Phosphorene: From theory to applications. Nat. Rev. Mater. 2016, 1, 16061-16069. [CrossRef]

10. Sorkin, V.; Cai, Y.; Ong, Z.; Zhang, G.; Zhang, Y.W. Recent advances in the study of phosphorene and its nanostructures. Crit. Rev. Solid State Mater. Sci. 2017, 42, 1-82. [CrossRef]

11. Cai, Y.; Zhang, G.; Zhang, Y.-W. (Eds.) Phosphorene: Physical Properties, Synthesis, and Fabrication; Jenny Stanford Publishing Pte. Ltd.: Singapore, 2019.

12. Goswami, A.; Gawande, M.B. Phosphorene: Current status, challenges and opportunities. Front. Chem. Sci. Eng. 2019, 13, 296-309. [CrossRef]

13. Bridgman, P.W. Two new modifications of Phosphorus. J. Am. Chem. Soc. 1914, 36, 1344-1363. [CrossRef]

14. Hultgren, R.; Gingrich, N.S.; Warren, B.E. The atomic distribution in red and black phosphorus and the crystal structure of black phosphorus. J. Chem. Phys. 1935, 3, 351-355. [CrossRef]

15. Brown, A.; Rundqvist, S. Refinement of the crystal structure of black phosphorus. Acta Cryst. 1965, 19, 684-685. [CrossRef]

16. Akhtar, M.; Anderson, G.; Zhao, R.; Alruqi, A.; Mroczkowska, J.E.; Sumanasekera, G.; Jasinsk, J.B. Recent advances in synthesis, properties, and applications of phosphorene. NPJ 2D Mater. Appl. 2017, 1, 5. [CrossRef]

17. Hu, Z.; Niu, T.; Guo, R.; Zhang, J.; Lai, M.; He, J.; Wang, L.; Chen, W. Two-dimensional black phosphorus: Its fabrication, functionalization and applications. Nanoscale 2018, 10, 21575-21603. [CrossRef]

18. Martini, F.; Borsacchi, S.; Barcaro, G.; Caporali, M.; Vanni, M.; Serrano-Ruiz, M.; Geppi, M.; Peruzzini, M.; Calucci, L. Phosphorene and black phosphorus: The 31P NMR view. J. Phys. Chem. Lett. 2019, 10, 5122-5127. [CrossRef]

19. Guan, L.; Xing, B.; Niu, X.; Wang, D.; Yu, Y.; Zhang, S.; Yan, X.; Wang, Y.; Sha, J. Metal-assisted exfoliation of few-layer black phosphorus with high yield. Chem. Commun. 2018, 54, 595-598. [CrossRef]

20. Han, Z.J.; Murdock, A.T.; Seo, D.H.; Bendavid, A. Recent progress in plasma-assisted synthesis and modification of 2D materials. 2D Mater. 2018, 5, 32002-32028. [CrossRef]

21. Lu, W.; Nan, H.; Hong, J.; Chen, Y.; Zhu, C.; Liang, Z.; Ma, X.; Ni, Z.; Jin, C.; Zhang, Z. Lasma-assisted fabrication of monolayer phosphorene and its Raman characterization. Nano Res. 2014, 7, 853-859. [CrossRef]

22. Pei, J.; Gai, X.; Yang, J.; Wang, X.; Yu, Z.; Choi, D.-Y.; Luther-Davies, B.; Lu, Y. Producing air-stable monolayers of phosphorene and their defect engineering. Nat. Commun. 2016, 7, 10450-10458. [CrossRef] [PubMed]

23. Kuriakose, S.; Ahmed, T.; Balendhran, S.; Collis, G.E.; Bansal, V.; Aharonovich, I.; Sriram, S.; Bhaskaran, M.; Walia, S. Effects of plasma-treatment on the electrical and optoelectronic properties of layered black phosphorus. Appl. Mater. Today 2018, 12, 244-249. [CrossRef]

24. Huang, H.; Gao, M.; Kang, Y.; Li, J.; Wang, J.; Wu, L.; Chu, P.K.; Huang, Y.; Ibarra, M.R.; Yu, X.-F. Rapid and scalable production of high-quality phosphorene by plasma-liquid technology. Chem. Commun. 2020, 56, 221-224. [CrossRef]

25. Yan, Z.; He, X.; She, L.; Sun, J.; Jiang, R.; Xu, H.; Shi, F.; Lei, Z.; Liu, Z.-H. Solvothermal-assisted liquid-phase exfoliation of large size and high quality black phosphorus. J. Mater. 2018, 4, 129-134. [CrossRef]

26. Gómez-Pérez, J.; Kónya, Z.; Kukovecz, A. Acetone improves the topographical homogeneity of liquid phase exfoliated few-layer black phosphorus flakes. Nanotechnology 2018, 29, 365303-365313. [CrossRef] [PubMed] 
27. Dhanabalan, S.C.; Ponraj, J.S.; Guo, Z.; Li, S.; Bao, Q.; Zhang, H. Emerging trends in phosphorene fabrication towards next generation devices. Adv. Sci. 2017, 4, 1600305-1600337. [CrossRef] [PubMed]

28. Liu, W.; Zhu, Y.; Chen, Z.; Lei, J.; Feng, P. Multilayer black phosphorus exfoliated with the aid of sodium hydroxide: An improvement in electrochemical energy storage. J. Electron. Mater. 2018, 47, 4793-4798. [CrossRef]

29. Watts, M.C.; Picco, L.; Russell-Pavier, F.S.; Cullen, P.L.; Miller, T.S.; Bartuś, S.P.; Payton, O.D.; Skipper, N.T.; Tileli, V.; Howard, C.A. Production of phosphorene nanoribbons. Nature 2019, 568, 216-220. [CrossRef] [PubMed]

30. Ambrosi, A.; Sofer, Z.; Pumera, M. Electrochemical exfoliation of layered black phosphorus into phosphorene. Angew. Chem. Int. Ed. 2017, 56, 10443-10445. [CrossRef]

31. Liu, N.; Kim, P.; Kim, J.H.; Ye, J.H.; Kim, S.; Lee, C.J. Large-area atomically thin $\mathrm{MoS}_{2}$ nanosheets prepared using electrochemical exfoliation. ACS Nano 2014, 8, 6902-6910. [CrossRef]

32. Zhao, M.; Guo, X.Y.; Ambacher, O.; Nebel, C.E.; Hoffmann, R. Electrochemical generation of hydrogenated graphene flakes. Carbon 2015, 83, 128-135. [CrossRef]

33. Wang, J.; Manga, K.K.; Bao, Q.; Loh, K.P. High-yield synthesis of few-layer graphene flakes through electrochemical expansion of graphite in propylene carbonate electrolyte. J. Am. Chem. Soc. 2011, 133, 8888-8891. [CrossRef]

34. Xiao, H.; Zhao, M.; Zhang, J.; Ma, X.; Zhang, J.; Hu, T.; Tang, T.; Jia, J.; Wu, H. Electrochemical cathode exfoliation of bulky black phosphorus into few-layer phosphorene nanosheets. Electrochem. Commun. 2018, 89, 10-13. [CrossRef]

35. Li, J.; Chen, C.; Liu, S.; Lu, J.; Goh, W.P.; Fang, H.; Qiu, Z.; Tian, B.; Chen, Z.; Yao, C.; et al. Ultrafast electrochemical expansion of black phosphorus toward high-yield synthesis of few-layer phosphorene. Chem. Mater. 2018, 30, 2742-2749. [CrossRef]

36. Luo, F.; Wang, D.; Zhang, J.; Li, X.; Liu, D.; Li, H.; Lu, M.; Xie, X.; Huang, L.; Haung, W. Ultrafast cathodic exfoliation of few-layer black phosphorus in aqueous solution. ACS Appl. Nano Mater. 2019, 2, 3793-3801. [CrossRef]

37. Liu, H.; Lian, P.; Zhang, Q.; Yang, Y.; Mei, Y. The preparation of holey phosphorene by electrochemical assistance. Electrochem. Commun. 2019, 98, 124-128. [CrossRef]

38. Khandelwal, A.; Mani, K.; Karigerasi, M.H.; Lahiri, I. Phophorene-The two dimensional black phosphorous: Properties, synthesis and applictions. Mater. Sci. Eng. B 2017, 221, 17-34. [CrossRef]

39. Zhang, J.L.; Zhao, S.; Han, C.; Wang, Z.; Zhong, S.; Sun, S.; Guo, R.; Zhou, X.; Gu, C.D.; Yuan, K.D.; et al. Epitaxial growth of single layer blue phosphorus: A new phase of two-dimensional phosphorus. Nano Lett. 2016, 168, 4903-4908. [CrossRef]

40. Gao, J.; Zhang, G.; Zhang, Y.-W. The critical role of substrate in stabilizing phosphorene nanoflake: A theoretical exploration. J. Am. Chem. Soc. 2016, 138, 4763-4771. [CrossRef]

41. Tian, B.; Tian, B.; Smith, B.; Scott, M.C.; Lei, Q.; Hua, R.; Tian, Y.; Liu, Y. Facile bottom-up synthesis of partially oxidized black phosphorus nanoshets as metal-free photocatalyst for hydrogen evolution. Proc. Natl. Acad. Sci. USA 2018, 115, 4345-4350. [CrossRef]

42. Xu, H.; Han, X.; Li, Z.; Liu, W.; Li, X.; Wu, J.; Guo, Z.; Liu, H. Epitaxial growth of few-layer black phosphorene quantum dots on Si substrates. Adv. Mater. Interfaces 2018, 5, 1801048-1801056. [CrossRef]

43. Sang, D.K.; Wang, H.; Guo, Z.; Xie, N.; Zhang, H. Recent developments in stability and passivation techniques of phosphorene toward next-generation device applications. Adv. Funct. Mater. 2019, 29, 1903419-1903431. [CrossRef]

44. Zhou, Q.; Chen, Q.; Tong, Y.; Wang, J. Light-induced ambient degradation of Fe-layer black phosphorus: Mechanism and protection. Angew. Chem. Int. Ed. 2016, 55, 11437-11441. [CrossRef] [PubMed]

45. Jain, R.; Singh, Y.; Cho, S.-Y.; Sasikala, S.P.; Koo, S.H.; Narayan, R.; Jung, H.-T.; Jung, Y.; Kim, S.O. Ambient stabilization of few layer phosphorene via noncovalent functionalization with surfactants: Systematic 2D NMR characterization in aqueous dispersion. Chem. Mater. 2019, 31, 2786-2794. [CrossRef]

46. Gusmão, R.; Sofer, Z.; Pumera, M. Functional protection of exfoliated black phosphorus by noncovalent modification with anthraquinone. ACS Nano 2018, 12, 5666-5673. [CrossRef] [PubMed]

47. Ghambarian, M.; Azizi, Z.; Ghashghaee, M. Functionalization and doping of black phosphorus. In Black Phosphorus; Inamuddin, I., Boddula, R., Asiri, A., Eds.; Engineering Materials; Springer: Cham, Switzerland, 2020; pp. 1-30. 
48. Kumar, A. Simultaneous passivation and encapsulation of black phosphorus nanosheets (phosphorene) by optically active polypeptide micelles for biosensors. ACS Appl. Nano Mater. 2019, 2, 2397-2404. [CrossRef]

49. Liang, S.; Wu, L.; Liu, H.; Li, J.; Chen, M.; Zhang, M. Organic molecular passivation of phosporene: An aptamer-based biosensing platform. Biosens. Bioelectron. 2019, 126, 30-35. [CrossRef]

50. Bolognesi, M.; Moschetto, S.; Trapani, M.; Prescimone, F.; Ferroni, C.; Manca, G.; Ienco, A.; Borsacchi, S.; Caporali, M.; Muccini, M.; et al. Noncovalent functionalization of 2D black phosphorus with fluorescent boronic derivatives of pyrene for probing and modulating the interaction with molecular oxygen. ACS Appl. Mater. Interfaces 2019, 11, 22637-22647. [CrossRef]

51. Qiu, M.; Ren, W.X.; Jeong, T.; Won, M.; Park, G.Y.; Sang, D.K.; Liu, L.-P.; Zhang, H.; Kim, J.S. Omnipotent phosphorene: A next-generation, two-dimensional nanoplatform for multidisciplinary biomedical applications. Chem. Soc. Rev. 2018, 47, 5588-5601. [CrossRef]

52. Luo, M.; Fan, T.; Zhou, Y.; Zhang, H.; Mei, L. 2D black phosphorus-based biomedical applications. Adv. Funct. Mater. 2019, 29, 1808306-1808325. [CrossRef]

53. Tatullo, M.; Genovese, F.; Aiello, E.; Amantea, M.; Makeeva, I.; Zavan, B.; Rengo, S.; Fortunato, L. Phosphorene is the new graphene in biomedical applications. Materials 2019, 12, 2301. [CrossRef]

54. Anju, S.; Ashtami, J.; Mohanan, P.V. Black phosphorus, a prospective graphene substitute for biomedical applications. Mater. Sci. Eng. C 2019, 97, 978-993.

55. Choi, J.R.; Yong, K.W.; Choi, J.Y.; Nilghaz, A.; Lin, Y.; Xu, J.; Lu, X. Black phosphorus and its biomedical applications. Theranostics 2018, 8, 1005-1026. [CrossRef] [PubMed]

56. Qu, G.; Xia, T.; Zhou, W.; Zhang, X.; Zhang, H.; Hu, L.; Shi, J.; Yu, X.-F.; Jiang, G. Property-activity relationship of black phosphorus at the nano-bio interface: From molecules to organisms. Chem. Rev. 2020, 120, $2288-2346$.

57. Sun, Z.; Zhang, Y.; Yu, H.; Yan, C.; Liu, Y.; Hong, S.; Tao, H.; Robertson, A.W.; Wang, Z.; Pádua, A.A.H. New solvent-stabilized few-layer black phosphorus for antibacterial applications. Nanoscale 2018, 10, 12543-12553. [CrossRef] [PubMed]

58. Mayorga-Martinez, C.C.; Gusmão, R.; Sofer, Z.; Pumera, M. Pnictogen-based enzymatic phenol biosensors: Phosphorene, arsenene, antimonene, and bismuthene. Angew. Chem. Int. Ed. 2019, 58, 134-138. [CrossRef]

59. Mayorga-Martinez, C.C.; Sofer, Z.; Pumera, M. Binary phosphorene redox behavior in oxidoreductase enzymatic systems. ACS Nano 2019, 13, 13217-13224. [PubMed]

60. Liang, H.; Xu, H.; Zhao, Y.; Zheng, J.; Zhao, H.; Li, G.; Li, C.-P. Ultrasensitive electrochemical sensor for prostate specific antigen detection with a phosphorene platform and magnetic covalent organic framework signal amplifier. Biosens. Bioelectron. 2019, 144, 111691. [CrossRef] [PubMed]

61. Li, P.; Chen, W.; Liu, D.; Huang, H.; Dan, K.; Hu, X.; Yu, S.; Chu, P.K.; Yu, X.-F. Template growth of $\mathrm{Au} / \mathrm{Ag}$ nanocomposites on phosphorene for sensitive SERS detection of pesticides. Nanotechnology 2019, 30, 275604-275612. [CrossRef]

62. Comber, S.; Gardner, M.; Georges, K.; Blackwood, D.; Gilmour, D. Domestic source of phosphorus to sewage treatment works. Environ. Technol. 2013, 34, 1349-1358. [CrossRef]

63. Huang, K.; Wu, J.; Gu, Z. Black phosphorus hydrogel scaffolds enhance bone regeneration via a sustained supply of calcium-free phosphorus. ACS Appl. Mater. Interfaces 2018, 11, 2908-2916.

64. Shao, J.; Ruan, C.; Xie, H.; Li, Z.; Wang, H.; Chu, P.K.; Yu, X.-F. Black-phosphorus-incorporated hydrogel as a sprayable and biodegradable photothermal platform for postsurgical treatment of cancer. Adv. Sci. 2018, 5, 1700848-1700855. [CrossRef] [PubMed]

65. Liu, G.; Tsai, H.-I.; Zeng, X.; Qi, J.; Luo, M.; Wang, X.; Mei, L.; Deng, W. Black phosphorus nanosheets-based stable drug delivery system via drug-self-stabilization for combined photothermal and chemo cancer therapy. Chem. Eng. J. 2019, 375, 121917-121927. [CrossRef]

66. Zong, S.; Wang, L.; Yang, Z.; Wang, H.; Wang, Z.; Cui, Y. Black phosphorus-based drug nanocarrier for targeted and synergetic chemophotothermal therapy of acute lymphoblastic leukemia. ACS Appl. Mater. Interfaces 2019, 11, 5896-5902. [CrossRef] [PubMed]

(C) 2020 by the authors. Licensee MDPI, Basel, Switzerland. This article is an open access article distributed under the terms and conditions of the Creative Commons Attribution (CC BY) license (http://creativecommons.org/licenses/by/4.0/). 